\title{
Moisture-retentive dressings: A review of the current literature
}

\author{
Duncan Nickerson BSc \\ Calgary, Alberta \\ Arnis Freiberg MD FRCSC FACS \\ Division of Plastic Surgery, Toronto Hospital, Western Division, Toronto, Ontario
}

\begin{abstract}
D Nickerson, A Freiberg. Moisture-retentive dressings: A review of the current literature. Can J Plast Surg 1995;3(1):3538. Moisture-retentive dressings allow rapid epithelialization, reduce pain, reduce fibrosis, reduce infection and yield better cosmetic results. Furthermore, occlusive dressings allow less painful debridement and stimulate granulation tissue. Despite these advantages, moisture-retentive dressings are still underused. Among the reasons for this underuse are confusion due to the variety of dressings available, fear of infection, and excessive cost. The studies reviewed here refute concerns that moisture-retentive dressings are associated with more infections or that they are too expensive to be practical. The issue of cost was addressed in several of the articles reviewed here, and while moisture-retentive dressings are certainly more expensive on a per unit basis, their overall cost-effectiveness can be more favourable than other more conventional dressings.
\end{abstract}

Key Words: Acute partial thickness wounds, Moisture-retentive dressings, Pressure sores, Venous stasis ulcers

\begin{abstract}
Pansements qui emprisonnent l'humidité : survol de la littérature actuelle
RÉSUMÉ : Les pansements qui emprisonnent l'humidité permettent une épithélialisation rapide, atténuent la douleur, réduisent la fibrose et l'infection et génèrent de meilleurs résultats sur le plan esthétique. De plus, les pansements occlusifs permettent un débridement moins douloureux et stimulent les tissus de granulation. Malgré ces avantages, ces pansements sont encore sousutilisés. Parmi les raisons de cette sous-utilisation, notons une certaine confusion attribuable à la variété des pansements disponibles, la peur de l'infection et leur coût élevé. Les études passées en revue ici réfutent les arguments à l'effet que les pansements qui emprisonnent l'humidité sont associés à un plus grand nombre d'infections ou qu'ils sont trop chers pour être pratiques. L'aspect économique a été étudié dans plusieurs articles passés en revue ici et, bien que ces pansements soient certainement plus chers par unité, leur rentabilité globale peut être plus favorable que d'autres pansements de type plus conventionnel.
\end{abstract}

A $\mathrm{n}$ ideal dressing performs the protective function of the epidermis while the underlying epithelial layer regenerates. At one time, dogma held that this was best accomplished by keeping wounds dry to avoid infection. However, there is now ample evidence that retaining the wound exudate in contact with the wound, rather than wicking it away, may promote healing. It is now widely recognized that moisture is key to wound healing (1-3). In the last decade or so, moisture-retentive dressings (MRD) which retain wound exudate have gained increasing acceptance.

\section{DEFINITION}

The types of dressings that can be categorized as being moisture-retentive are listed in Table 1.

Motta, in reviewing moisture-retentive dressings (2) also includes calcium alginate exudate absorbers such as Kaltostat (Calgon Vestal, St Louis, Missouri) and Sorbsan (PharmaPlast Ltd, Worcestershire, England) which, while capable of absorbing large quantities of exudate (up to 20 times their own weight), do not desiccate the wound.

Correspondence and reprints: Duncan Nickerson, 11 Edenwold Place, Calgary, Alberta T3A 3T7. Telephone (403) 239-4167.
The term 'occlusive dressing' is often used to describe this class of dressings, but this term is not entirely accurate, since different dressings within the broad category of MRD

\section{TABLE 1: Types of moisture retentive dressings}

\section{Films}

-thin, transparent, adherent polyurethane

-permeable to water vapour, $\mathrm{O}_{2}, \mathrm{CO}_{2}$

-prototype: OpSite ${ }^{\circ}$ (Smith Nephew, Lachine, Quebec)

-mainly useful in superficial wounds

\section{Foams}

-nonadherent polyurethane, taped in place over the wound -permeable to water vapour, $\mathrm{O}_{2}, \mathrm{CO}_{2}$

-prototype: Synthaderm (Lock Laboratories, Kent, England)

-useful when there is a dead space to be packed

\section{Hydrocolloids}

-absorbent polyurethane

-water and gas impermeable (a true 'occlusive dressing')

-prototype: Duoderm (ConvaTec, Montreal, Quebec)

-useful in heavily exudative wounds

\section{Hydrogels}

-polyethylene oxide membrane

-high water content and thus very soothing

-prototype: Vigilon (Bard Home Health, Murray Hill, New Jersey)

-also useful in heavily exudative wounds 
are in fact permeable to different substances, while retaining varying degrees of wound exudate next to the wound.

\section{MECHANISM OF ACTION}

The most widely accepted explanation for a moist environment favouring healing involves the exposure of the wound surface to stimulatory molecules (4-6). Research has demonstrated that fluid from occluded wounds stimulates growth of fibroblasts, keratinocytes, and endothelial cells, as well as glycosaminoglycan production in vitro $(4,5)$. The validity of the association between the action of the wound fluid and the role of growth factors therein is strengthened by the observation that these effects are blocked if antibodies to platelet-derived growth factor are added to the culture media (5). Other growth factors present in wound fluid include IL-1, EGF, and TGF-B (4).

Furthermore MRD, by creating a moist environment around the wound, prevent crust formation over the wound. A moist wound without a crust resurfaces $40 \%$ faster than an air-exposed, crusted wound (5). This wound crust has been postulated to act as a physical impediment to epithelialization; this theory, however, lacks experimental validation (6).

Calcium alginate works as follows: alginic acid consists of the 1,4 beta isomer of mannuronic acid and the 1,4 alpha isomer of glucuronic acid; in fact, it is closely related to cellulose except that carboxyl moieties are substituted for the alcohol groups of cellulose. These carboxyl groups react with ammonium or metallic salts to form corresponding alginate salts. When calcium alginate reacts with body fluids calcium is exchanged for sodium, rendering the alginate soluble in aqueous media. As a result, the product forms a gel which is in turn broken down to simple glucose-like monomers which are absorbed by the tissue (7).

\section{INDICATIONS}

The scenarios for use of different moisture-retentive dressings are reviewed in depth by both Motta and Eaglstein $(2,6)$. Films (eg, OpSite) are useful mainly for superficial, minimally exudative wounds. If a wound is heavily exudative, hydrocolloids (eg, DuoDERM), hydrogels (eg, Vigilon), or alginate preparations (eg, Kaltostat) are all options. Alginates and foams become particularly useful when heavy exudate is encountered in the context of a wound with dead space or undermining, in order to fill the defect at the same time as absorbing the exudate.

\section{CONTRAINDICATIONS}

It was a long-held doctrine that wounds should be kept dry to avoid infection. Initial reaction to MRD was cautious because it was felt that the moisture would predispose to infection. This fear of infection with moisture-retentive dressings has largely been refuted. Eaglstein (6) cites the relative impermeability of moisture-retentive dressings to exogenous bacteria, the ability of accumulated neutrophils in the wound fluid to destroy bacteria, and the reduced amount of necrotic material available in occluded wounds as factors which actually lead to fewer infections in wounds treated occlusively. In the case of one MRD (DuoDERM), Wyatt (8) points out that bacterial growth in vitro is retarded at the acidic $\mathrm{pH}$ that is typically encountered in a DuoDERM-dressed wound. Having said that, MRD should never be applied to a wound that is already clinically infected or where colonization by anaerobes is suspected.

Due to their relative impermeability, it is widely recognized $(2,5,6,9,10)$ that moisture-retentive dressings are contraindicated in grossly infected wounds. It must also be recognized that because they trap wound fluid and promote exudation, moisture-retentive dressings will often develop a malodorous yellow gel beneath them which should not be confused with infection $(2,4-6,9,10)$. It is actually beneficial to continue with the moisture-retentive dressing when this gel is present, since in addition to necrotic cell debris and liquefied eschar it also contains growth factors and enzymes which promote autolysis and healing $(6,11)$.

Mulder et al (11) report a small clinical series in which they make observations regarding apparent fibrinolytic properties of three different hydrocolloid dressings (DuoDERM, Restore (Hollister Inc, Libertyville, Illinois) and Comfeel Ulcus (Coloplast Inc, Tampa, Florida) (11). Whereas clots were found after Restore and Comfeel Ulcus were used, no clots were observed under DuoDERM. This would seem to suggest that DuoDERM is contraindicated as a hemostatic dressing to an acute wound in a vascular area, eg, the scalp.

\section{CLINICAL EXPERIENCES}

\section{Acute, partial thickness wounds}

Tan et al (12) used donor sites of 71 patients who had split thickness skin grafts harvested for a variety of indications to evaluate DuoDERM versus scarlet red as a dressing for partial thickness wounds (scarlet red, a nonocclusive gauze impregnated with an azo dye, promotes epithelialization). Ninety percent of donor sites treated with DuoDERM had healed at 10 days, as compared with $57 \%$ of the scarlet redtreated donor sites. Scarlet red was also responsible for more complaints of discomfort with dressing removal than DuoDERM. On the negative side of the ledger, the incidence of leakage with DuoDERM was 20 times higher (assessed as number of dressing changes per wound). This inevitably led to DuoDERM being 7.59 times more expensive than scarlet red for a 10-day treatment period.

Tan and colleagues used the same model to compare DuoDERM with Zenoderm (Zenith Technology Co, New Zealand), a semi-occlusive hydrogel which allows water vapour loss (13). DuoDERM again surpassed the other dressing for healing at 10 days: $97 \%$ versus $75 \%$ for Zenoderm. However, the practical advantage of this in the long term may be outweighed by the finding that all donor sites in both groups had healed by day 15 and that DuoDERM leaked twice as much as Zenoderm and cost 2.25 times more. Furthermore, patients reported no difference in pain with dressing removal.

Lawrence et al have compared calcium alginate with scarlet red as a dressing for 46 split thickness skin graft donor sites (7). Each site was located on the thigh, and their ap- 
proach was to dress half of each donor site with calcium alginate and half with scarlet red. Their finding was that at 10 days an average of $72 \%$ of the surface area dressed with calcium alginate had healed as compared to $84 \%$ with scarlet red. These results may lack validity since it is conceivable that a bias could occur if one of the dressings had more absorbency than the other and thus contributed indirectly to the healing of the other half of the wound by lateral absorption. Calcium alginate, at least anecdotally (2), is more absorbent than scarlet red and so in a sense may have been doing the work of both dressings, artificially prolonging the healing of the side that it was applied to. In addition the poor performance of calcium alginate may be a tradeoff to the decreased pain reported elsewhere with calcium alginate (2).

Smith et al conducted a prospective clinical trial in 30 patients who had split thickness skin grafts harvested (14). Donor sites were randomized to treatment with either DuoDERM or fine mesh gauze. By eight days, $33 \%$ of wounds treated with DuoDERM had healed, compared to only $8 \%$ for fine mesh gauze. At 17 days, the figures were $83 \%$ and $62 \%$, respectively. Clinical infection was present in fully $25 \%$ of the gauze-dressed wounds but in none of the DuoDERM group. The authors made no comment as to cost.

A study by Madden et al (4) randomized 58 patients to treatment with either DuoDERM or one of three control dressings. The three control dressings used were: OpSite, silver sulfadiazine, or fine mesh gauze. Time to total healing with DuoDERM was $60.3 \%$ of that with gauze and $66.7 \%$ of that with silver sulfadiazine. There was no statistically significant difference in healing times between DuoDERM and OpSite. By extension then, both of these occlusive dressings outperformed silver sulfadiazine and gauze. As an adjunct to their study, the authors harvested exudate from the DuoDERM- and OpSite-occluded wounds and found that it stimulated the growth of keratinocytes in culture, lending support to the concept that the mechanism of action by which occlusive dressings promoted epithelialization is by exposing the wound to endogenous growth factors.

Wyatt and his colleagues have also compared DuoDERM to silver sulfadiazine for managing partial thickness wounds (8). Twenty-five patients were randomized to each treatment group with the outcome being that DuoDERM was subjectively found to be associated with less pain, better compliance and better aesthetic acceptance. Objectively, healing was assessed by the number of days to healing, with DuoDERM healing significantly faster. DuoDERM was also less expensive over the complete healing course. While initial cost was high, treatment came to a successful end sooner, leading to a net saving: silver sulfadiazine ultimately proved to cost 2.3 times as much as a course of DuoDERM. Furthermore, silver has systemic complications which negatively impact the healing process. These include neutropenia and inhibition of protein and DNA synthesis.

\section{Pressure sores}

Others have shown hydrocolloid MRD to be an effective choice in pressure sores. Xakellis and Chrischilles (15) set out with the express purpose of determining the costeffectiveness of hydrocolloid dressings versus saline-soaked gauze dressings for pressure ulcers. While the cost per dressing change was 3.3 times higher with the hydrocolloid dressing, the nursing time required for a saline-soaked gauze dressing change was eight times higher than that for a hydrocolloid dressing change. After factoring in the nursing wages, this translated to a $37 \%$ saving with hydrocolloid dressings. There was no significant difference in healing times between the two treatments.

Colwell et al (1) have also compared the efficacy and cost-effectiveness of saline-moistened gauze with hydrocolloid wafers in managing pressure ulcers. They found that healing was slower with gauze, that infection rates were higher, and that gauze cost 3.45 times more than hydrocolloid in the long term because healing times were longer, dressing changes took more time, and more dressing changes were required. Furthermore, they found that hydrocolloid wafers were met with better patient acceptance since they were less painful to change than gauze dressings.

\section{Venous stasis ulcers}

Two dressings commonly used for treatment of venous stasis ulcers are DuoDERM, a moisture-retentive hydrocolloid, and the so-called 'Unna's boot'. Unna's paste consists of a moist paste impregnated with zinc oxide, calamine lotion and glycerin. It is applied as a 'cast' and provides topical treatment and compression and is changed weekly (16). Several authors have compared the traditional Unna's boot with hydrocolloid dressings such as DuoDERM.

Kikta et al (16) compared the Unna's boot to DuoDERM in 1988. Sixty-nine venous stasis ulcers were followed until healing or six months, whichever endpoint was attained first. Seventy percent of the ulcers in the Unna's boot group were healed at six months, compared with $38 \%$ in the DuoDERM group. Among the ulcers that did heal, there was no statistical significance in the time to healing between the two groups. It is important to point out, as the authors did, that this is perhaps an unfair comparison since a compressive dressing was not applied over the DuoDERM, yielding an uncontrolled variable that may have played a role in the superior performance of the Unna's boot. Of interest is that DuoDERM was met with better patient acceptance.

The shortcoming of the above study was addressed in 1992 by Cordts et al (17), who compared Unna's boot to DuoDERM used in combination with a compressive dressing. Healing rates were three times faster with DuoDERM, as assessed by wound planimetry over the first four weeks of healing. At 12 weeks, the rate was still greater with DuoDERM but was no longer statistically significant.

Two papers were found which compared hydrocolloid treatment of venous stasis ulcers to treatments other than Unna's boot $(18,19)$. Friedman et al $(18)$ randomized 20 ulcers to treatment with either a hydrocolloid dressing or gauze soaked in Sweitzer's solution (an antimicrobial solution comprising $1 \%$ copper sulphate, $2 \%$ zinc sulphate and camphor water). All 20 ulcers healed, and there was no signifi- 
cant difference in time to healing. It was, however, noted that patients reported less pain with the hydrocolloid dressing.

Backhouse et al (19) randomized 56 patients with chronic venous ulcers to treatment with either a hydrocolloid (Granuflex, UK marketing name for DuoDERM) or a porous, nonocclusive dressing. Measuring outcome in terms of number of ulcers healed in each group at six and at 12 weeks, the authors found no difference between the two treatment groups. Indeed, $75 \%$ of ulcers in each group ultimately went on to heal, leading the authors to recognize that there were other factors in both groups that favoured healing. Both groups received compression dressings in addition to the underlying dressing and it was a conclusion of this study that compression played a key role in healing, irrespective of the underlying dressing.

\section{SUMMARY}

In his recent review, Eaglstein (6) supports the contention that in the acute wound, moisture-retentive dressings allow rapid epithelialization, reduce pain, reduce fibrosis, reduce infection and yield better cosmetic results. Furthermore, he

\section{REFERENCES}

1. Colwell JC, Foreman MD, Trotter JP. A comparison of the efficacy and cost-effectiveness of two methods of managing pressure ulcers. Decubitus 1993;6(4):28-36.

2. Motta GJ. Dressed for success: How moisture-retentive dressings promote healing. Nursing 1993;December:26-33.

3. Hunt TK, Mueller RV, Goodson, WH III. Wound Healing. In: Current Surgical Diagnosis and Treatment. Norwalk: Lange, 1994:89-90.

4. Madden MR, Nolan E, Finkelstein JL, Yurt RW, et al. Comparison of an occlusive and a semi-occlusive dressing and the effect of the wound exudate upon keratinocyte proliferation. J Trauma 1989;29:924-31.

5. Falanga V. Occlusive wound dressings: why, when, which? Arch Dermatol 1988;124:872-7.

6. Eaglstein WH. Occlusive dressings. J Dermatol Surg Oncol 1993;19:716-20.

7. Lawrence JE, Blake GB. A comparison of calcium alginate and scarlet red dressings in the healing of split thickness skin graft donor sites. $\mathrm{Br} \mathrm{J}$ Plast Surg 1991;44:247-9.

8. Wyatt D, McGowan DN, Najarian MP. Comparison of a hydrocolloid dressing and silver sulfadiazine cream in the outpatient management of second-degree burns. J Trauma 1990;30:857-65.

9. Barnes HR. Alternating transparent and hydrocolloid dressings: a difficult case. Nursing 1993;March:59-61.

10. Fowler E, Cuzzell JZ, Papen JC. Healing with hydrocolloid. Am J Nursing 1991;February:63-4. argues that occlusive dressings allow less painful debridement and stimulate granulation tissue. Despite these advantages, Eaglstein contends that moisture-retentive dressings are still underused. Among the reasons that he postulates for this underuse are confusion due to the variety of dressings available, fear of infection and excessive cost.

The studies reviewed here refute concerns that moistureretentive dressings are associated with more infections or that they are too expensive to be practical. The issue of cost was addressed in several of the articles reviewed here, and while moisture-retentive dressings are certainly more expensive on a per unit basis, their overall cost-effectiveness can be more favourable than other more conventional dressings $(8,15)$.

Perhaps the most sensible comment on the use of moisture-retentive dressings has been made by Falanga (5), when he pointed out "the dressing is actually a pharmacologic agent, a drug with its own benefits and risks". Indeed, as several of the articles reviewed displayed, these dressings can be very beneficial in some settings, and patient acceptance is generally excellent.

11. Mulder GD, Walker A. Preliminary observations on clotting under three hydrocolloid dressings. J Roy Soc Med 1989;82:739-40.

12. Tan ST, Roberts RH, Blake GB. Comparing DuoDERM E with scarlet red in the treatment of split skin graft donor sites. Br J Plast Surg 1993;46:79-81.

13. Tan ST, Roberts RH, Sinclair SW. A comparison of Zenoderm with DuoDERM E in the treatment of split skin graft donor sites. Br J Plast Surg 1993; 46:82-4.

14. Smith DJ, Thomson PD, Bolton LL, Hutchinson JJ. Microbiology and healing of the occluded skin-graft donor site. Plast Reconstr Surg 1993;91:1094-7.

15. Xakellis GC, Chrischilles EA. Hydrocolloid versus saline-gauze dressings in treating pressure ulcers: a cost-effectiveness analysis. Arch Phys Med Rehab 1992;73:463-9.

16. Kikta MJ, Schuler JJ, Meyer JP, et al. A prospective, randomized trial of Unna's boots versus hydroactive dressing in the treatment of venous stasis ulcers. J Vasc Surg 1988;7:478-86.

17. Cordts PR, Hanrahan LM, Rodriguez AA, Woodson J, Lamorte WW, Menzoian JO. A prospective, randomized trial of Unna's boot versus DuoDERM CGF hydroactive dressing plus compression in the management of venous leg ulcers. J Vasc Surg 1992;15:480-6.

18. Friedman SJ, Su WDP. Management of leg ulcers with hydrocolloid occlusive dressing. Arch Dermatol 1984;120:1329-36.

19. Backhouse CM, Blair SD, Savage AP, Walton J, McCollum CN. Controlled trial of occlusive dressings in healing chronic venous ulcers. Br J Surg 1987;74:626-7. 